\title{
A Polyhedral Approach for a Constrained Matching Problem*
}

\author{
A. Hefner ${ }^{1}$ and P. Kleinschmidt ${ }^{2}$ \\ ${ }^{1}$ Corporate Research and Development (ZFE T SE 1), Siemens AG, \\ 81730 München, Germany \\ Andreas.Hefner@zfe.siemens.de \\ ${ }^{2}$ Department of Business Administration and Economics, University of Passau, \\ Innstraße 29, 94032 Passau, Germany \\ kleinsch@winf.uni-passau.de
}

\begin{abstract}
Certain manpower scheduling problems can be solved as weighted matching problems with additional constraints. A complete linear description of a polytope whose vertices correspond to such matchings is provided and how this can be used for solving more complex problems with a cutting plane procedure is described.
\end{abstract}

\section{Introduction}

The matching problem and its special case, the assignment problem, are well-studied problems in combinatorial optimization for which efficient, i.e., polynomial-time, algorithms exist.

Matching problems are often mentioned in the context of manpower scheduling where people have to be assigned to jobs. However, matching problems in their pure form can hardly be applied in this area because in practice many additional constraints have to be satisfied.

In this paper we describe two types of such constraints which we were faced with in a printing plant and we demonstrate a polyhedral approach for solving such problems.

Before describing the application we introduce the problems in a formal way.

Let $G=(V, E)$ be an undirected graph with edge weights $c_{e} \in \mathbb{Z}_{+}$for each $e \in E$. A matching $M$ of $G$ is a subset of the edges such that no two edges share the same

\footnotetext{
* This research was supported by Grant 03-KL7PAS-6 of the German Federal Ministry of Research and Technology.
} 


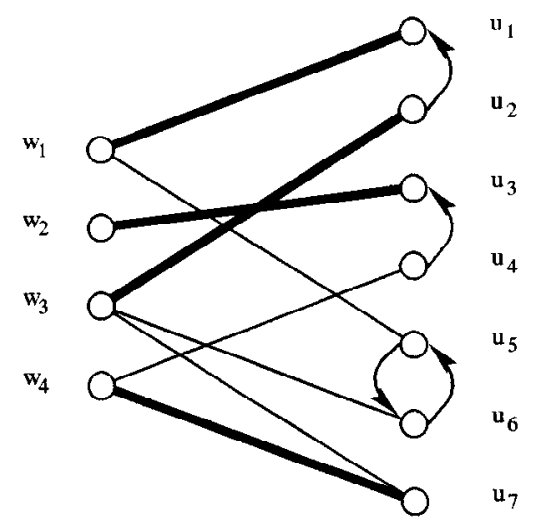

Fig. 1. An MS-matching.

node. The (weighted) matching problem is the problem of finding a matching $M$ such that $\sum_{e \in M} c_{e}$ is maximum.

Let $D=(V, A)$ be a digraph with the same node set as $G$. We call $D$ a dependence digraph of $G$. If $(u, v)$ is an arc in $A$, we say that $u$ is a slave of $v$ and that $v$ is a master of $u$. The maximum size of a connected component of $D$ is denoted by $k(D)$.

A Master/Slave-matching (MS-matching for short) of $G$ with respect to $D$ is a matching $M$ of $G$ with the property that if $(u, v) \in A$ and $u$ is matched, then so is $v$ (see Fig. 1 for an example of an MS-matching). If the dependence digraph $D$ is clear from the context, we simply say that $M$ is an $M S$-matching of $G$ instead of saying that $M$ is an $M S$-matching of $G$ with respect to $D$.

The (weighted) MS-matching problem is the problem of finding an MS-matching $M$ of $G$ such that $\sum_{e \in M} c_{e}$ is maximum. If $k(D)=2$ we call the problem 2-MS-matching.

We introduce some further notation for use in later sections. Let $G=(V, E)$ be a graph. A node $v \in V$ which is not incident to any edge is called isolated. The graph $G$ is bipartite if the node set $V$ can be partitioned into two disjoint sets, $W$ and $U$, such that each edge has one node in $W$ and the other in $U$. The sets $W$ and $U$ are the color classes of $G$ and $W \cup U$ is the bipartition of $G$. Let $U_{1}, \ldots, U_{m}$ be pairwise disjoint subsets of $U$ and let $r_{1}, \ldots, r_{m}$ be positive integers. The capacity-constrained matching problem (CCMP) is to find a matching $M$ of $G$ with maximum weight and the additional property that $M$ matches at most $r_{i}$ nodes from $U_{i}, 1 \leq i \leq m$.

The MS-matching problem arises in our application in the following context. People with different skills who have to handle printing presses of different types are employed in the printing plant. A worker who is qualified to operate a certain printing machine is called an operator (for this machine). For some presses it is possible to reduce the runthrough time of tasks by assigning an assistant to the operator. The assistant relieves the operator of a press in his work, e.g., by inserting new paper in the printing press, but he cannot handle the press on his own. In general, each worker is qualified to perform some of the operator jobs and some of the assistant jobs. The printing presses can be distinguished by the number and kind of people who operate them: there are presses which can be handled by a single operator (type 1), presses which can be handled by an operator who may be supported by an assistant (type 2), and presses which require 
the simultaneous service of two operators (type 3). The problem is to design an optimal manpower schedule, i.e., to assign the workers to the printing presses such that the following two conditions are satisfied: if some worker is assigned to the assistant job of a machine of type 2, then some other worker must be assigned to the operator job of the same machine, too. For a machine of type 3 either both jobs are filled or none of them is. Furthermore, the assignment should be optimal with respect to the different qualifications of the workers and the requirements of the jobs.

We can model this situation as an MS-matching problem. We construct a bipartite graph $G=(V, E)$ with bipartition $V=W \cup U$ and a dependence digraph $D=(V, A)$. The nodes in $W$ correspond to workers and the nodes in $U$ correspond to jobs. For each machine of type 1 there is a single node in $U$. For each machine of type 2, $U$ contains a node $u$ associated with the operator job and a node $u^{\prime}$ associated with the assistant job and $A$ contains the arc $\left(u^{\prime}, u\right)$. Finally, for each machine of type 3, $U$ has two nodes $u$, $u^{\prime}$ associated with the two operator jobs, and $A$ contains the two $\operatorname{arcs}\left(u, u^{\prime}\right)$ and $\left(u^{\prime}, u\right)$ (consider again Fig. 1 for an example of this model: the nodes $\left\{u_{1}, u_{2}\right\}$ model a machine of type $2,\left\{u_{5}, u_{6}\right\}$ a machine of type 3 , and node $u_{7}$ a machine of type 1$)$. For two nodes $w \in W$ and $u \in U$ the edge $[w, u]$ is included in $E$ if the worker corresponding to $w$ is qualified to do the job corresponding to $u$. The edge weight $c_{w u}$ measures how well the worker is trained for the job. A large edge weight value means a better qualification of the worker for the job. As all components of $D$ have at most two nodes in this model we have a 2-MS-matching problem.

CCMP comes up in a model involving different shifts. The printing plant is operating three shifts. Based on data from production planning it is desired to fill some jobs only in one or two shifts. A priori it is not decided in what shift(s) such a job is not filled. Fixing shifts for jobs explicitly gives less flexibility and hence worse schedules. We leave it to a CCMP to find optimal shift assignments. A set $U_{i}, 1 \leq i \leq m$, in the definition of CCMP corresponds to several copies of the same job-node, one for every shift which is feasible for that job. The bound $r_{i}$ is the maximum number of shifts in which this job should be filled. This means that at most $r_{i}$ nodes from $U_{i}$ should be matched.

In our application we had to deal with the constraints of 2-MS-matching and CCMP simultaneously. We call the corresponding problem the capacity-constrained 2-MSmatching problem (CC2MSM).

The statements of the following theorem have been proved in $[\mathrm{H}]$ and $[\mathrm{HK}]$.

\section{Theorem 1.}

(i) MS-matching is NP-complete (even if $k(D)=3$ ).

(ii) 2-MS-matching can be solved in $O\left(|V|^{3}\right)$ time.

(iii) CCMP can be transformed to the min-cost-flow problem.

(iv) CC2MSM is NP-complete.

While (ii) and (iii) say that our individual problems are easy, (iv) says that their combination is a hard problem. As we had to solve it anyway, we studied its polyhedral structure in order to obtain a basis for solving CC2MSM by a branch-and-cut procedure. The advantage of this approach is the flexibility of adding even more constraints to the problem if the application requires this (it does). 
In Section 2 we prove a complete description of the polytope whose vertices are the 2-MS-matchings of a given graph.

In Section 3 we show how this description can be used to solve CC2MSM with branch and cut and we present some practical experience.

\section{The Polytope $\operatorname{MSM}(G, D)$}

Let $G$ and $D$ be the graph and the digraph in an instance of MS-matching and let $k(D)=2$, i.e., $G$ and $D$ describe the feasible set of a 2-MS-matching problem. The polytope $\operatorname{MSM}(G, D)$ is defined as the convex hull of the incidence vectors of all MSmatchings of $G$ with respect to $D$.

We need some notation for formulating our results for $\operatorname{MSM}(G, D)$.

For a node $v \in V$ let $\delta(v)$ denote the set of all $e \in E$ which are incident with $v$. For $V_{1}, V_{2} \subset V$ let $\delta\left(V_{1}, V_{2}\right)$ denote the set of edges having one node in $V_{1}$ and one in $V_{2}$. The set $E\left(V_{1}\right)$ is the set of edges having both nodes in $V_{1}$.

Let $x \in \mathbb{R}^{E}$ be a vector indexed by the elements of $E$ and let $x_{e}$ denote the coordinate of $x$ corresponding to $e \in E$. i.e.,

For $F \subset E$ define $x(F):=\sum_{e \in F} x_{e}$ and let $\chi^{F} \in \mathbb{R}^{E}$ be the incidence vector of $F$,

$$
\chi_{e}^{F}:= \begin{cases}1, & e \in F \\ 0, & e \notin F\end{cases}
$$

Then we define

$$
\operatorname{MSM}(G, D):=\operatorname{conv}\left\{\chi^{M} \mid M \text { is an MS-matching in } G \text { with respect to } D\right\} .
$$

$\operatorname{MSM}(G, D)$ is a polytope in $\mathbb{R}^{E}$ whose vertices are precisely the incidence vectors of the MS-matchings of $G$ with respect to $D$. We define a partition $V:=V_{F} \cup V_{M} \cup V_{C} \cup V_{S}$ of our node set as follows: $V_{F}$ is the set of nodes which are not incident with any arc of $D$. For nodes $v$ and $v^{\prime}$ with $\left(v, v^{\prime}\right) \in A$ and $\left(v^{\prime}, v\right) \in A$ we put one node, say $v$, into the set $V_{M}$ and the other one into the set $V_{C}$. If $\left(v, v^{\prime}\right) \in A$ but $\left(v^{\prime}, v\right) \notin A$ we let $v \in V_{S}$ and $v^{\prime} \in V_{M}$. As $D$ has no components with more than two nodes this clearly defines a partition of $V$.

Let $f: V \rightarrow V$ be the partial injective function defined by $f(v)=v^{\prime}$ if and only if $\left(v, v^{\prime}\right) \in A$.

Our main result is the following theorem which describes the polytope $\operatorname{MSM}(G, D)$ in terms of equalities and inequalities.

Theorem 2. $\operatorname{MSM}(G, D)$ is precisely the set of all $x \in \mathbb{R}^{E}$ which satisfy the following constraints:

$$
\begin{aligned}
& x_{e} \geq 0 \quad \text { forall } e \in E, \\
& x(\delta(v)) \leq 1 \quad \text { for all } v \in V_{F} \cup V_{M}, \\
& x(\delta(v))-x(\delta(f(v)))=0 \quad \text { for all } \quad v \in V_{C} \text {, } \\
& x(\delta(v))-x(\delta(f(v))) \leq 0 \quad \text { for all } v \in V_{S} \text {, }
\end{aligned}
$$




$$
\begin{aligned}
& x(E(S \backslash T))-x(E(T))-x(\delta(T, V \backslash S)) \leq \frac{|S|-1}{2}-|T| \\
& \quad \text { for all } \quad S \subseteq V, \quad|S| \geq 3, \quad|S| \text { odd, } \quad T=\left\{v \in S \cap V_{M} \mid f^{-1}(v) \in S\right\} .
\end{aligned}
$$

Proof. Let $P$ be the polytope given by (1)-(5). We have to show that $P=\operatorname{MSM}(G, D)$.

It is easy to verify that the integer points satisfying (1)-(4) are precisely the incidence vectors of the 2-MS-matchings. Conditions (1) and (2) are the conditions for $x$ to be a matching and (3) and (4) correspond to the dependencies defined by $D$. To show $P \supseteq \operatorname{MSM}(G, D)$ it thus suffices that (5) is valid for every $x \in M S M(G, D)$. We in fact show that the inequalities (5) are Chvátal-Gomory-Cuts with respect to (1)-(4) and they have Chvátal-rank 1.

Let $S$ and $T$ be as defined in the theorem and set $\bar{T}:=\left\{v \in V_{M} \backslash S \mid f^{-1}(v) \in S\right\}$. For all nodes $v \in(S \backslash T) \cup \bar{T}$ we add the corresponding constraint from (2)-(4) and for every edge from $\delta(S, V \backslash S)$ we subtract the inequality (1). This yields

$$
\begin{aligned}
& \sum_{v \in S \backslash T} x(\delta(v))-\sum_{v \in S \cap\left(V_{C} \cup V_{S}\right)} x(\delta(f(v)))+\sum_{v \in \bar{T}} x(\delta(v))-x(\delta(S, V \backslash S)) \\
&=\sum_{v \in S \backslash T} x(\delta(v))-\sum_{v \in T} x(\delta(v))-\sum_{v \in \bar{T}} x(\delta(v))+\sum_{v \in \bar{T}} x(\delta(v))-x(\delta(S, V \backslash S)) \\
&= 2 x(E(S \backslash T))+x(\delta(S \backslash T))-2 x(E(T))-x(\delta(T))-x(\delta(S, V \backslash S)) \\
&= 2 x(E(S \backslash T))+x(\delta(S \backslash T, T))+x(\delta(S \backslash T, V \backslash S))-2 x(E(T)) \\
&-x(\delta(S \backslash T, T))-x(\delta(V \backslash S, T))-x(\delta(S \backslash T, V \backslash S))-x(\delta(T, V \backslash S)) \\
&= 2 x(E(S \backslash T))-2 x(E(T))-2 x(\delta(T, V \backslash S)) \\
& \leq\left|(S \backslash T) \cap\left(V_{F} \cup V_{M}\right)\right|+|\bar{T}| \\
&=\left|S \cap V_{F}\right|+\left|S \cap V_{M}\right|-|T|+\left|S \cap\left(V_{C} \cup V_{S}\right)\right|-\left|f^{-1}(T)\right| \\
&=|S|-2|T| .
\end{aligned}
$$

Dividing this inequality by 2 and rounding yields (5). It remains to show that $P \subseteq$ $\operatorname{MSM}(G, D)$. For every $\bar{x} \in P$ we show that it is a convex combination of MS-matchings of $G$. For this we define an auxiliary graph $H=(V, F)$ as follows:

$F$ contains all edges in $E$ and in addition for every $v \in V_{M}$ it contains an artificial edge joining $v$ and $f^{-1}(v)$.

Observe that $H$ may contain parallel edges. It will always be clear from the context whether we deal with edges of $E$ or artificial edges.

Let

$$
Q:=\operatorname{conv}\left\{\chi^{M} \mid M \text { is a matching of } H \text { which matches every node in } V_{M} \cup V_{C}\right\} \text {. }
$$

In [HK], Lemma 3(a), we have proved that a matching $M^{\prime}$ of $H$ corresponding to a vertex of $Q$ has the property that $M^{\prime} \cap E$ is a 2-MS-matching of $G$ with respect to $D$. We exploit this strong connection of matchings of $H$ and MS-matchings of $G$ for our proof by using the fact that a complete description of $Q$ follows directly from Edmonds' complete description of the matching-polytope [E]. 
This description of $Q$ is as follows:

$$
\begin{aligned}
x_{e} & \geq 0 & & \text { for all } \quad e \in F, \\
x\left(\delta_{H}(v)\right) & =1 & & \text { for all } \quad v \in V_{M} \cup V_{C}, \\
x\left(\delta_{H}(v)\right) & \leq 1 & & \text { for all } \quad v \in V_{F} \cup V_{S}, \\
x\left(E_{H}(S)\right) & \leq \frac{|S|-1}{2} & & \text { for all } \quad S \subset V, \quad|S| \geq 3, \quad \text { and } \quad|S| \text { odd } .
\end{aligned}
$$

The index $H$ in the symbols $\delta_{H}$ and $E_{H}$ indicates that we are referring to the edges of $H$. For our given $\bar{x} \in P$ we define some $x^{\prime} \in \mathbb{R}^{F}$ which will turn out to be an element of $Q$.

We set $x_{e}^{\prime}=\bar{x}_{e}$ for all $e \in E$ and $x_{\left[f^{-1}(v), v\right]}^{\prime}=1-\bar{x}\left(\delta_{G}(v)\right)$ for all $v \in V_{M}$. From the definition of $x^{\prime}$ the nonnegativity conditions (6) follow immediately.

For $v \in V_{M}$ we have

$$
x^{\prime}\left(\delta_{H}(v)\right)=x^{\prime}\left(\delta_{G}(v)\right)+x_{\left[f^{-1}(v), v\right]}^{\prime}=\bar{x}\left(\delta_{G}(v)\right)+1-\bar{x}\left(\delta_{G}(v)\right)=1
$$

and for $v \in V_{C}$ we have

$$
x^{\prime}\left(\delta_{H}(v)\right)=x^{\prime}\left(\delta_{G}(v)\right)+x_{[v, f(v)]}^{\prime}=\bar{x}\left(\delta_{G}(v)\right)+1-\bar{x}\left(\delta_{G}(f(v))\right)=1,
$$

where the last equation follows from (3). This implies that $x^{\prime}$ satisfies (7).

The validity of (8) is proved as follows. For $v \in V_{F}$ we have $x^{\prime}\left(\delta_{H}(v)\right)=x^{\prime}\left(\delta_{G}(v)\right)=$ $\bar{x}\left(\delta_{G}(v)\right) \leq 1$ where the last inequality follows from (2). For $v \in V_{S}$ we have $x^{\prime}\left(\delta_{H}(v)\right)=$ $x^{\prime}\left(\delta_{G}(v)\right)+x_{[v, f(v)]}^{\prime}=\bar{x}\left(\delta_{G}(v)\right)+1-\bar{x}\left(\delta_{G}(f(v))\right) \leq 1$, where the last inequality follows from (4).

The blossom inequalities (9) for $x^{\prime}$ follow from

$$
\begin{aligned}
x^{\prime}\left(E_{H}(S)\right)= & x^{\prime}\left(E_{G}(S)\right)+\sum_{v \in T} x_{f^{-1}(v) v}^{\prime} \\
= & \bar{x}\left(E_{G}(S)\right)+\sum_{v \in T}\left(1-\bar{x}\left(\delta_{G}(v)\right)\right) \\
= & \bar{x}\left(E_{G}(S)\right)+|T|-2 \bar{x}\left(E_{G}(T)\right)-\bar{x}\left(\delta_{G}(T)\right) \\
= & \bar{x}\left(E_{G}(T)\right)+\bar{x}\left(E_{G}(S \backslash T)\right)+\bar{x}\left(\delta_{G}(T, S \backslash T)\right) \\
& +|T|-2 \bar{x}\left(E_{G}(T)\right)-\bar{x}\left(\delta_{G}(T, S \backslash T)\right)-\bar{x}\left(\delta_{G}(T, V \backslash S)\right) \\
= & \bar{x}\left(E_{G}(S \backslash T)\right)-\bar{x}\left(E_{G}(T)\right)-\bar{x}\left(\delta_{G}(T, V \backslash S)\right)+|T| \\
\leq & \frac{|S|-1}{2},
\end{aligned}
$$

where the last inequality follows from (5).

We have proved that $x^{\prime} \in Q$ and hence there exist $d \leq \operatorname{dim}(Q)+1$ matchings 


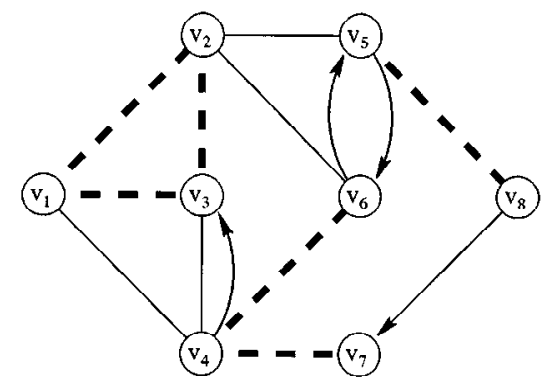

Fig. 2. Broken edges correspond to variables with value $\frac{1}{2}$, the other edges correspond to variables with value 0 .

$M_{1}^{\prime}, \ldots, M_{d}^{\prime}$ of $H$, all of which match every node of $V_{M} \cup V_{C}$ and there exist coefficients $\lambda_{1}, \ldots, \lambda_{d} \geq 0$ such that $\sum_{i=1}^{d} \lambda_{i}=1$ and $\sum_{i=1}^{d} \lambda_{i} \chi^{M_{i}^{\prime}}=x^{\prime}$.

We have remarked before that for every such $M_{i}^{\prime}$ the set $M_{i}^{\prime} \cap E$ is an MS-matching of $G$. This implies $\sum_{i=1}^{d} \lambda_{i} \chi^{M_{i}^{\prime} \cap E}=x_{E}^{\prime}=\bar{x}$ and hence $\bar{x} \in M S M(G, D)$. This completes the proof.

If $T=\emptyset$ the inequalities (5) become the classical blossom inequalities (9). Hence our description of the MS-matching-polytope is a proper generalization of Edmonds' description of the matching polytope. We call the inequalities (5) MS-blossom inequalities.

The MS-blossom inequalities are necessary for a description of $\operatorname{MSM}(G, D)$. The set of points defined by (1)-(4) may have fractional vertices as demonstrated by the example of Fig. 2.

In Fig. 2 we have an undirected graph $G$ whose dependency graph $D$ has four arcs. The vector $\bar{x}$ having value $\frac{1}{2}$ for the broken edges of $G$ and value 0 for the other edges satisfies (1)-(4). It is in fact a vertex of this system because it is the unique LP-maximum for the objective function $x_{12}+x_{13}-x_{14}+x_{23}-x_{25}-x_{26}-x_{34}+x_{46}$.

The vector $\bar{x}$ violates the MS-blossom inequality for $S=\left\{v_{4}, v_{5}, v_{6}, v_{7}, v_{8}\right\}$ and $T=$ $\left\{v_{5}, v_{7}\right\}$. This inequality is $x_{46}-x_{25} \leq 0$. It also violates the inequality $x_{12}+x_{13}+x_{23} \leq 1$ which is a classical blossom inequality $\left(S=\left\{v_{1}, v_{2}, v_{3}\right\}, T=\emptyset\right)$.

In [HK] we have used the auxiliary graph $H$ for a transformation of 2-MS-matching to ordinary weighted matching. This yields the $O\left(|V|^{3}\right)$ efficient algorithm for 2-MSmatching mentioned in Theorem 1(ii).

Of course, Theorem 2 implies that 2-MS-matching can be solved by the LP defined by (1)-(5). However, in general there are exponentially many MS-blossom inequalities which make it impossible even to formulate the LP efficiently already for smaller instances. This is not a real problem in this case because-as in the case of the matching problem - the LP could be solved with the primal-dual method in which only a polynomial number of MS-blossom inequalities are actually used.

Another possibility for solving 2-MS-matching is to use Theorem 2 as a basis for a cutting plane approach. This is the subject of the next section. 


\section{A Cutting Plane Approach for CC2MSM}

As mentioned in the Introduction we have to solve 2-MS-matching with additional constraints, e.g., the problem CC2MSM which is NP-complete. In [H] we have also considered team-oriented constraints and situations where MS-matching problems have a dependence digraph $D$ with $k(D) \geq 3$.

For all those problems the inequalities (1)-(5) are still valid (for MS-matching problems with $k(D) \geq 3$ the MS-blossom inequalities are only valid for maximal subgraphs $D^{\prime}$ of $D$ with $\left.k\left(D^{\prime}\right) \leq 2\right)$.

As there are too many MS-blossom inequalities, it therefore makes sense to use them only as cutting planes in a branch-and-cut algorithm (see [GL] for more on branch-andcut algorithms).

In such an algorithm we use an LP-relaxation for our problem consisting of the constraints (1)-(4) and a reasonably small set of other constraints. If the LP relaxation has an integer optimal solution we have solved our problem. Otherwise, we determine valid constraints which are violated by a fractional solution $x$ of the relaxation. If we can identify such a constraint, we add it to the LP and resolve it. If we cannot identify such a constraint, we may have to branch on fractional variables.

We used the MS-blossom inequalities (5) as cutting planes for our problems because identifying a violated one - the separation problem—can be solved in polynomial time. The separation problem for the ordinary blossom inequalities (9) in a matching problem can be transformed to the problem of finding a minimum odd cut in a graph. An algorithm of Padberg and Rao [PR] solves this problem in polynomial time. For the separation problem of the MS-blossom inequalities we can use this algorithm, too. We basically have to use the same idea as in the proof of Theorem 2: transform the MS-matching problem for $G$ to a matching problem for the auxiliary graph $H$, use the Padberg-Rao algorithm on the auxiliary problem and translate it back to the original problem (for details see $[\mathrm{H}])$.

It turned out that this procedure is quite successful for CC2MSM occurring in manpower scheduling. The LP relaxation of CC2MSM uses the constraints (1)-(4) and the capacity constraints for the sets $U_{i}$ with their capacities $r_{i}$ :

$$
x\left(\delta\left(U_{i}\right)\right) \leq r_{i}, \quad 1 \leq i \leq m .
$$

The integral points which satisfy (1)-(4) and the capacity constraints are of course exactly the feasible points of CC2MSM.

We applied the above procedure to several test problems, which we have got from the printing plant. In the largest real-world problem, which was available to us, 53 workers had to be assigned to 73 jobs, of which 46 were involved in MS constraints and 18 in capacity constraints. We were able to solve all problems of such a size on a SUN SPARCstation 10 within one CPU second. As the LP solver we used CPLEX 2.1. On average we could fill $4 \%$ more jobs in the printing plant than human operators. This figure may not look spectacular but considering the costs of human resources and machines it still represents a substantial increase in productivity.

The computing times for our real-world instances were always on a level which did not affect the interactivity of a system which we have developed for manpower scheduling. 


\section{References}

[E] J. Edmonds: Paths, trees and flowers, Canadian Journal of Mathematics 17 (1965), 449-467.

[GL] M. Grötschel, L. Lovász: Combinatorial optimization, in: R. L. Graham, M. Grötschel, L. Lovász (eds.), Handbook of Combinatorics, vol. 2, Elsevier, Amsterdam, 1995, pp. 1541-1597.

[H] A. Hefner: Polyedrische Methoden zur Lösung von Matching-Problemen mit Zusatzbedingungen, Dissertation, Universität Passau, 1995.

[HK] A. Hefner, P. Kleinschmidt: A constrained matching problem, Annals of Operations Research 57 (1995), 135-145.

[PR] M. W. Padberg, M. R. Rao: Odd minimum cut-sets and b-matchings, Mathematics of Operations Research 7 (1982), 67-80.

Received April 3, 1996, and in revised form July 9, 1996. 